\title{
Arousal and Stress Effects on Consolidation and Reconsolidation of Recognition Memory
}

\author{
Mouna Maroun' and Irit Akirav*,2 \\ 'Department of Neurobiology and Etiology, The Brain and Behavior Research Center, University of Haifa, Haifa, Israel; ${ }^{2}$ Department \\ of Psychology, The Brain and Behavior Research Center, University of Haifa, Haifa, Israel
}

\begin{abstract}
This study examined the effects of the arousal level of the rat and exposure to a behavioral stressor on consolidation and reconsolidation of a nonaversive learning paradigm, the object recognition task. Learning was tested under two arousal conditions: no previous habituation to the experimental context (high novelty stress/arousal level) or extensive prior habituation (reduced novelty stress/arousal level). Results indicated that in the habituated rats, exposure to an out-of-context stressor (ie, elevated platform stress) impaired longterm consolidation and reconsolidation of object recognition. RU-486, a glucocorticoid receptor (GR) antagonist, infused into the basolateral amygdala (BLA), reversed the impairing effects of the stressor. In contrast, the nonhabituated aroused rats were impaired when consolidation was examined, but their memory was intact following reactivation of the memory trace. Exposure of nonhabituated rats to an out-of-context stressor enhanced the long-term consolidation of recognition memory, but impaired reconsolidation, and the effects were reversed by a GR antagonist infused into the BLA. Additionally, nonhabituated control rats showed intact retrieval following microinfusion of propranolol to the BLA immediately after the training, suggesting an involvement of beta-adrenoceptors in the BLA in the arousal-induced impairment of consolidation. These findings demonstrate opposite effects, detrimental and facilitative, of arousal and stress on memory consolidation and reconsolidation. In addition, the data suggests that although some general features underlie consolidation and reconsolidation, there is a possible dissimilarity between the two processes, which is dependent on the arousal level of the animal during training.
\end{abstract}

Neuropsychopharmacology (2008) 33, 394-405; doi: I0.1038/sj.npp. I 30 I40 I; published online II April 2007

Keywords: stress; basolateral amygdala; RU-486; glucocorticoids; beta-adrenoceptors; propranolol

\section{INTRODUCTION}

Exposure to stress, as well as the release of stress hormones, for example, epinephrine and glucocorticoids from the adrenal during or after emotionally arousing experiences plays a critical role in consolidating lasting memories (de Kloet et al, 1999; McGaugh, 2002; McIntyre et al, 2003; Roozendaal, 2002). Numerous studies found enhancing as well as impairing effects on learning and memory following acute stress or acute glucocorticoid treatment (Akirav et al, 2001; Diamond et al, 1996; de Kloet et al, 1999; Lupien and McEwen, 1997; Roozendaal, 2002; Sandi et al, 1997; Trneckova et al, 2005). Additionally, extensive evidence focuses on the role of the amygdala, and specifically the basolateral amygdala (BLA), as a critical system that mediates stress hormone effects on memory consolidation

\footnotetext{
*Correspondence: Dr I Akirav, Department of Psychology, The Brain and Behavior Research Center, University of Haifa, Haifa 31905, Israel, Tel: +972 4 8249657, Fax: +97248249157,

E-mail: iakirav@psy.haifa.ac.il

Received 7 November 2006; revised 5 February 2007; accepted 20 February 2007
}

(Akirav and Richter-Levin, 2002; McGaugh, 2002; Roozendaal, 2003).

Memory consolidation refers to the progressive postacquisition stabilization of the memory trace (Dudai, 2002). When a memory is reactivated, it must undergo additional consolidation (reconsolidation) so as to persist (Alberini, 2005; Eisenberg et al, 2003; Milekic and Alberini, 2002; Nader et al, 2000a, b; Sara, 2000). To determine whether reconsolidation recapitulates consolidation, several groups have recently investigated whether the same molecules and pathways mediate the formation of a memory and its maintenance after reactivation (Akirav and Maroun, 2006; Anokhin et al, 2002; Debiec et al, 2002; Kida et al, 2002; Lee et al, 2004; Nader et al, 2000a; Przybyslawski and Sara, 1997; Torras-Garcia et al, 2005).

Here we used a different approach and aimed to examine whether the arousal level of the animal or exposure to an out-of-context stressor would differentially affect consolidation and reconsolidation of a nonaversive paradigm, the object recognition task. In other studies comparing consolidation and reconsolidation, a reinforcing stimulus is usually present during the consolidation phase but absent in the reconsolidation phase, thereby confounding the interpretation of the comparison (Nader, 2003). The object 
recognition task, on the other hand, does not involve an explicit exogenous reinforcer in the consolidation or reconsolidation phases. Because no rewarding or aversive stimulation is used during training, the learning occurs under conditions of relatively low stress or arousal (Ennaceur and Delacour, 1988). Arousal is not a passive process and can be regulated by environmental and task demands (Robbins, 1997). Accordingly, the placement of rats into an unfamiliar testing apparatus does evoke some degree of novelty-induced arousal, and repeated habituation of rats to the experimental context is known to reduce this arousal response (Cerbone and Sadile, 1994; Okuda et al, 2004; Roozendaal et al, 2006). Thus, rats habituated to the training apparatus would be expected to be less aroused during the training than rats not given prior habituation training.

The present study investigated the effects of the arousal level of the animal and its interaction with an exposure to an out-of-context stressor on consolidation and reconsolidation of recognition memory. We present data to suggest that it is the arousal level of the rat during training that plays a role in dissociating consolidation from reconsolidation of recognition memory.

\section{MATERIALS AND METHODS}

\section{Animals}

Male Wistar rats ( $\sim 60$ day old, $250-300$ g) were caged individually at $22 \pm 2{ }^{\circ} \mathrm{C}$ under 12 -h light/dark cycles. All rats were allowed free access to food and water.

\section{Drugs}

The GR antagonist RU-38486 (10 ng/0.5 $\mu \mathrm{l})$ and the $\beta$ adrenergic antagonist propranolol (Prop, $0.75 \mu \mathrm{g} / 0.5 \mu \mathrm{l}$ ) were obtained from Sigma (St Louis, MO). RU-38486 was first dissolved in $100 \%$ ethanol and subsequently diluted in saline to reach the appropriate concentration. The final concentration of ethanol was $2 \%$. The RU-486 dose was based on previous work showing a significant effect of RU-486 on memory when microinfused into the BLA (Roozendaal and McGaugh, 1997a; Roozendaal et al, 1999). Controls were given the vehicle $(2 \%$ ethanol) only. Prop was dissolved in physiological saline, which was also used as a control.

\section{Surgery and Drug Administration}

Rats were anesthetized with $4.8 \mathrm{ml} / \mathrm{kg}$ equithesin $(2.12 \%$ (w/ v) $\mathrm{MgSO}_{4}, 10 \%(\mathrm{v} / \mathrm{v})$ ethanol, $39.1 \%(\mathrm{v} / \mathrm{v})$ propylene glycol, $0.98 \%(\mathrm{w} / \mathrm{v})$ sodium pentobarbital, and $4.2 \%(\mathrm{w} / \mathrm{v})$ chloral hydrate), restrained in a stereotactic apparatus (David Kopf Instruments, Tujunga, CA), and implanted bilaterally with a stainless steel guide cannula (23-gauge, thin wall) aimed to the BLA (anteroposterior, $-3 \mathrm{~mm}$ relative to bregma; lateral, $\pm 5 \mathrm{~mm}$; ventral, $-7.4 \mathrm{~mm}$ ). The cannulae were positioned in place with acrylic dental cement and secured by two skull screws. A stylus was placed in the guide cannula to prevent clogging. Animals were allowed 1 week to recuperate before being subjected to experimental manipulations.

For microinfusion, the stylus was removed from the guide cannula, and a 28-gauge injection cannula, extending
$1.0 \mathrm{~mm}$ from the tip of the guide cannula, was inserted. The injection cannula was connected via PE20 tubing to a Hamilton micro syringe driven by a microinfusion pump (CMA/100; Carnegie Medicin, Stockholm, Sweden). Microinfusion was performed bilaterally in a $0.5 \mu \mathrm{l}$ volume per side delivered over $2 \mathrm{~min}$. The injection cannula was left in position before withdrawal for an additional minute to minimize dragging of the injected liquid along the injection tract.

\section{Object Recognition Task}

Object recognition memory is the ability to discriminate the familiarity of previously encountered objects. It was tested in a paradigm based on spontaneous exploration behavior of the rat (Ennaceur and Delacour, 1988). If a rat is presented with both a familiar object and a novel object, it will direct more exploration at the novel object. The objects were located in squared black open field $(50 \times 50 \times 50 \mathrm{~cm})$ under dim light and were fixed to the floor of the open-field arena, $10 \mathrm{~cm}$ from the walls. The objects used in the present study were children's toys made of plastic and varied in shape and texture, and were chosen basis of weight and size, that is, they were no larger than the rats and were relatively immovable. From rat to rat, the role (familiar or new object) as well as the relative positions of the two objects were counterbalanced and randomly permuted. The open field and the objects were thoroughly cleaned between trials with odorous clean wipes.

Exploration was defined as sniffing or touching the object with the nose and/or forepaws. Turning around or sitting on the object is not considered as exploratory behavior. A discrimination index calculated for each animal was expressed as $T_{\mathrm{N}} /\left(T_{\mathrm{N}}+T_{\mathrm{F}}\right)\left(T_{\mathrm{F}}=\right.$ time spent exploring the familiar object; $T_{\mathrm{N}}=$ time spent exploring the novel object). The amount of time spent exploring each object was recorded by an observer who was blind to the treatment. Intact recognition memory in the test phase is reflected in a discrimination score higher than 0.5 , which implies greater exploration of the novel object.

\section{Habituation and Handling}

About half of the rats were habituated to the experimental apparatus by allowing them to explore it for 5 min twice a day for 4 days before the experiment was performed (habituated). No object was placed inside the arena during habituation. The other half was not habituated to the experimental apparatus, only handled once a day for 2 days in their home cage (nonhabituated).

It has been recently demonstrated in an object recognition task that animals that were not habituated to the experimental apparatus showed a twofold increase in plasma corticosterone levels as compared with habituated animals (Okuda et al, 2004). Furthermore, the nonhabituated animals in the present open-field test were found to exhibit a higher level of anxiety (see Results section).

\section{Consolidation Protocol}

In the sample phase (day 1), each rat was placed in the open-field arena and allowed to explore two different objects for 5 min (A and B). The test phase (day 2) was given 
$24 \mathrm{~h}$ after the sample trial. During the 5-min test trial, the rat was presented with a duplicate of an object from the sample trial and a novel object (A and C). Exposure to the stressor or microinfusion of vehicle, RU-486 or Prop, to the BLA took place immediately following the sample phase on day 1. All figures show discrimination index in the test phase.

\section{Reconsolidation Protocol}

In the sample phase (day 1), each rat was placed in the open-field arena and allowed to explore two different objects for $5 \mathrm{~min}$ (A and B). Twenty-four hours later (day 2), they were exposed to the same two sample objects for a 5 -min period in order to reactivate the memory trace (A and $B$ ). The test phase (day 3) was given $24 \mathrm{~h}$ after the reactivation trial. During the 5-min test trial, the rat was presented with a duplicate of an object from the sample/ reactivation trial and a novel object (A and $\mathrm{C}$ ). Exposure to the stressor or microinfusion of vehicle, RU-486 or Prop, to the BLA took place immediately following the reactivation phase on day 2. All figures show discrimination index in the test phase.

\section{Elevated Platform Stress}

In this behavioral stressor, the animals are placed on an elevated platform (EP; $12 \times 12 \mathrm{~cm}$ ) for $30 \mathrm{~min}$ in a brightly lit room (Xu et al, 1998). The rats exhibit behavioral 'freezing', that is, immobility for up to $10 \mathrm{~min}$, defecation, and urination. This stressor was found to increase plasma corticosterone levels by $38 \%$ as compared with naïve rats (Kavushansky and Richter-Levin, 2006). It has also recently been found to impair long-term potentiation in the CA1 area of the hippocampus and in the BLA-medial prefrontal pathway (Maroun and Richter-Levin, 2003).

\section{Open-Field Test}

The open field was located in a small, quiet room fitted with a video camera $25 \mathrm{~cm}$ above the apparatus. The rats were tested under a low-illumination red light to simulate darkness and minimize stress levels. The open-field consisted of a base and black walls $(50 \times 50 \times 50 \mathrm{~cm})$ divided into $25(5 \times 5)$ identical sectors by white stripes. The squares were subdivided into peripheral and central sectors, where the central sector included the nine central squares $(3 \times 3)$ and the peripheral sector contained the squares close to the wall. Both the habituated and the nonhabituated rats were placed in the central sector, and their activity was recorded for $5 \mathrm{~min}$ by a video camera and taped for further analysis. The open-field arena was thoroughly cleaned between each test using odorous clean wipes. Open-field activity was manually scored from a TV screen by an observer who was blind to the treatment. We quantified the percent time spent in the center and in the corners of the open-field arena.

In an open-field arena, rats prefer the periphery to the center of the apparatus. Entering the center, which assesses approach-avoidance toward novel stimuli, is considered a reliable index of anxiety; it responds to anxiolytic agents (Ramos et al, 1997) and is sensitive to stress-induced anxiety states (Pare, 1994; Izumi et al, 1997; Durand et al,
1999). Hence, higher anxiety corresponds to less entering the center of the arena.

\section{Histology}

At the end of the experiments, rats were anesthetized, and their brains were removed, frozen, sectioned, and inspected for cannula placement using Nissl staining. The locations of cannula tips for the drugs and saline groups are shown in Figure 1.

\section{Statistics}

Differences between the groups were determined using ANOVA and $t$-tests. All post hoc comparisons were made using the least significant difference multiple comparison test (LSD).

\section{Approval}

The experiments were approved by the Ethics and Animal Care Committee of the University of Haifa and were performed in accordance with the National Institute of Health Guidelines for the Care and Use of Laboratory Animals.

\section{RESULTS}

\section{Nonhabituated Rats Spend Less Time in the Center of the Open Field}

We assessed the level of arousal in habituated and nonhabituated rats using the open-field test. Nonhabituated rats spent significantly less time exploring the central areas as compared with the habituated rats $(t(10)=4.533$, $p<0.001 ; n=6$ each; Figure 2). This suggests higher anxiety levels in the nonhabituated rats compared with the habituated rats.

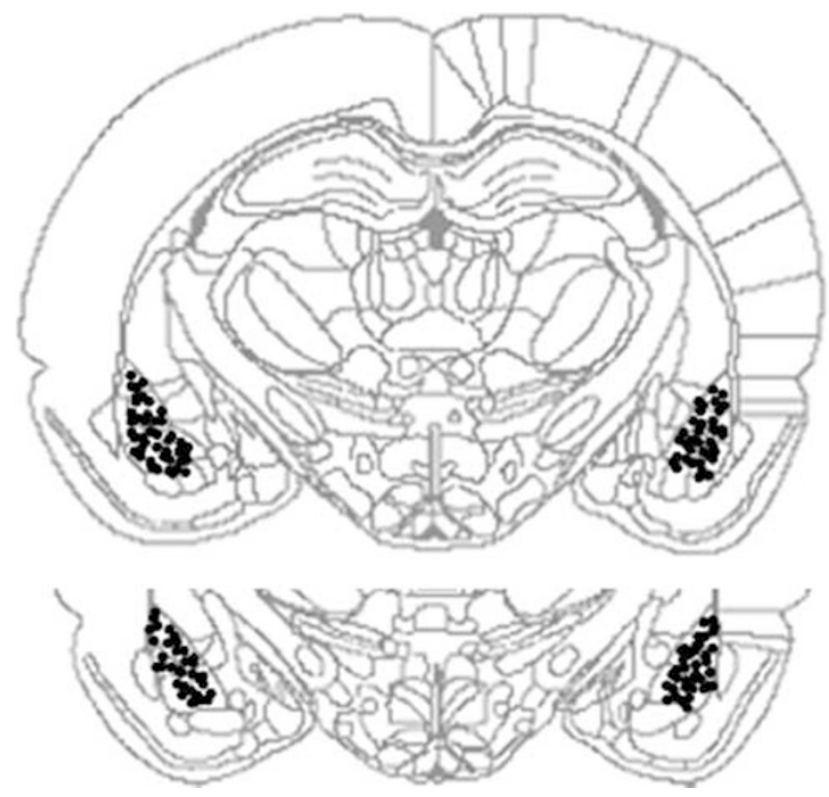

Figure I Schematic drawing of BLA cannulae tip positions. A coronal view is shown at position 3.14 and $3.30 \mathrm{~mm}$ posterior to bregma. 
Differential Performance of Habituated and Nonhabituated Rats in the Consolidation of Object Recognition

Eight groups of rats that were habituated to the experimental context before training (habituated) or just handled (nonhabituated) were exposed to the sample phase on day 1. Immediately afterwards, they were either microinfused with vehicle to the BLA and taken to their home cage (habituated cont, $n=10$; nonhabituated cont, $n=7$ ); exposed to the EP stress (habituated EP, $n=13$; nonhabituated EP, $n=7)$; microinfused with RU-486 and exposed to the EP (habituated RU + EP, $n=9$; nonhabituated RU + EP, $n=7$ ); or microinfused with RU-486 (habituated RU, $n=6$; nonhabituated RU, $n=5$ ), without exposure to the stressor (Figure 3a).

A three-factor ANOVA conducted on discrimination index on day 2 (arousal: habituated/nonhabituated; drug: RU-486/vehicle; stress: stress/no-stress) revealed a significant arousal, drug and stress $\left(\mathrm{F}_{(1,56)}=25.2, p<0.001\right)$ interaction, indicating that the pattern of the effects of stress and drug depended on the arousal level. There was a main effect of arousal $\left(\mathrm{F}_{(1,56)}=5.953, p=0.018\right)$, but not drug $\left(\mathrm{F}_{(1,56)}=1.756, \mathrm{NS}\right)$ or stress $\left(\mathrm{F}_{(1,56)}<1, \mathrm{NS}\right)$. The interactions between arousal and drug $\left(\mathrm{F}_{(1,56)}=24.964, p<0.001\right)$, arousal and stress $\left(\mathrm{F}_{(1,56)}=27.865, p<0.001\right)$, drug and stress $\left(\mathrm{F}_{(1,56)}=4.942, p=0.03\right)$ were all significant. Further analysis of the data showed a significant difference between the groups in discrimination index in the test on day 2 $(\mathrm{F}(7,56)=16.822, p<0.0001)$. Post hoc comparisons revealed that the stressed habituated group (EP) spent significantly less time exploring the new object as compared with the other habituated $(p<0.0001)$ and nonhabituated groups (cont: $p<0.01$; EP: $p<0.0001$; RU: $p=0.051$; $\mathrm{RU}+\mathrm{EP}: \quad p<0.001$ ) ( $t$-test for difference from 0.5 in the habituated group: cont: $t(9)=5.91, p<0.001$; RU: $t(5)=8.69, p<0.001$; RU + EP: $t(8)=3.628, p<0.01)$. Thus, exposure to stress impaired the consolidation of recognition memory in the habituated rats, and microinfusing RU-486 to the BLA reversed the impairment. Merely microinfusing RU-486 to the BLA in the nonstressed rats did not affect their performance. Post hoc comparisons also revealed that

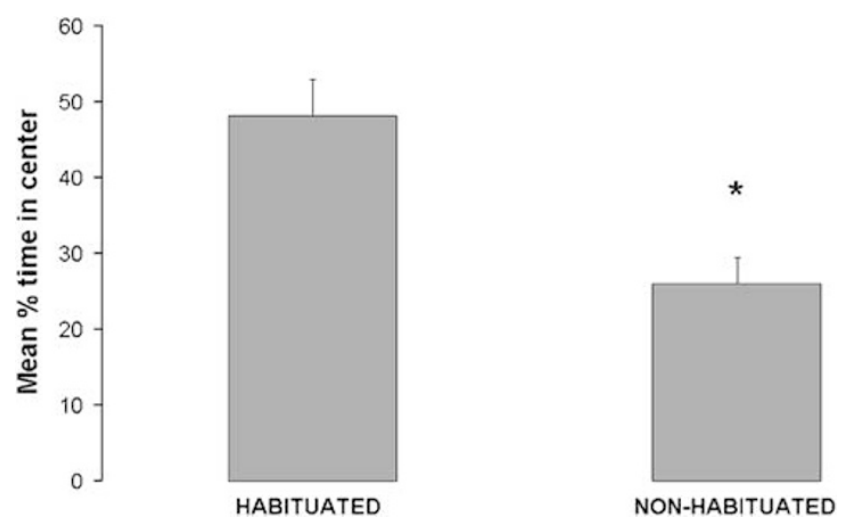

Figure 2 Nonhabituated rats spend less time in the center of the open field. Nonhabituated rats spent significantly less time exploring the central areas as compared with the habituated rats $(* p<0.00 \mathrm{I})$, suggesting higher anxiety levels in the nonhabituated rats compared with the habituated rats. the stressed nonhabituated group (EP) spent significantly more time exploring the new object as compared with the nonhabituated groups (cont and RU groups: $p<0.0001, \mathrm{RU}$ $+\mathrm{EP}: p<0.01)$ ( $t$-test for difference from 0.5 in the stressed nonhabituated group: $t(6)=5.153, p<0.01)$. Thus, in contrast to the habituated rats, the nonhabituated control rats showed impaired performance in the test, and exposure to stress enhanced the recognition memory consolidation. Microinfusing RU-486 to the BLA reversed the enhancement of the stressor and merely microinfusing RU-486 to the BLA in the nonstressed rats did not affect their performance. Further, there was no significant difference between the groups in discrimination index $(F(7,56)<1$, NS) during the sample phase (day 1; data not shown).

A significant difference in total exploration time was found between the groups during the test phase $(\mathrm{F}(7,56)=8.183, p<0.0001$; Table 1$)$. Post hoc comparisons revealed that the nonhabituated groups spent significantly more time exploring the new object compared with the habituated groups $(p<0.05)$. The difference in exploration time between habituated and nonhabituated animals is consistent with reports in the literature suggesting a
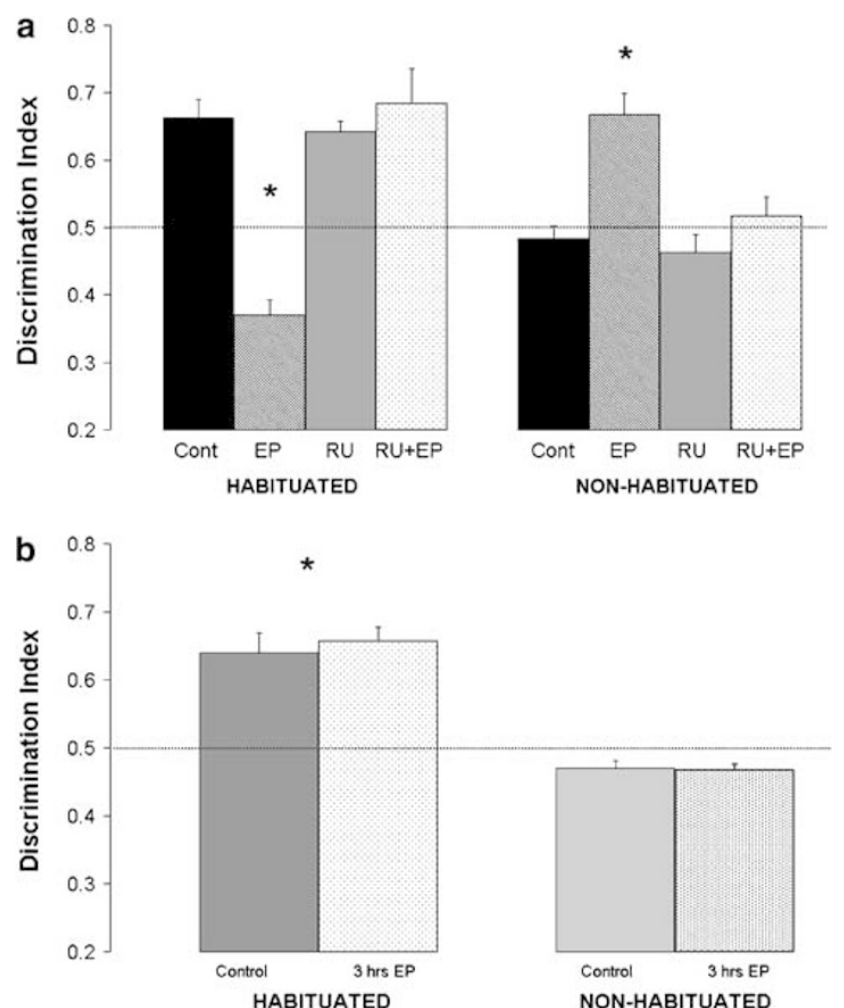

Figure 3 Differential performance of habituated and nonhabituated rats in the consolidation of object recognition. (a) Habituated rats that were exposed to the stressor after the sample phase showed impaired performance on day 2 compared with the other groups $(* p<0.001)$. Nonhabituated rats that were exposed to the stressor after the sample phase showed intact performance on day 2 compared with the other groups $(* p<0.001)$. RU-486 into the BLA reversed the impairing (habituated) and enhancing (nonhabituated) effects of the stressor. (b) Habituated and nonhabituated rats that were exposed to the stressor $3 \mathrm{~h}$ after the sample phase were no different from their control counterparts, that is, habituated animals showed intact performance in the test on day 2 and nonhabituated animals showed poor performance $(* p<0.001$ indicates a difference between habituated and nonhabituated groups). 
Table I Total Exploration Time in Habituated and Nonhabituated Animals

\begin{tabular}{llr}
\hline Figure \# & Group & Test exploration \\
\hline $3 a$ & Habituated-Cont & $25.54 \pm 1.85$ \\
& Habituated-EP & $26.19 \pm 1.20$ \\
& Habituated-RU & $30.20 \pm 4.89$ \\
& Habituated-RU+EP & $27.96 \pm 4.55$ \\
& Nonhabituated-Cont & $48.73 \pm 6.42$ \\
& Nonhabituated-EP & $39.65 \pm 4.16$ \\
& Nonhabituated-RU & $43.53 \pm 4.03$ \\
& Nonhabituated-RU+EP & $52.94 \pm 2.46$ \\
& Habituated-Cont & $39.19 \pm 3.97$ \\
Ha & Habituated-EP & $40.28 \pm 2.70$ \\
& Habituated-RU & $38.25 \pm 2.11$ \\
& Habituated-RU+EP & $45.76 \pm 5.11$ \\
& Nonhabituated-Cont & $58.17 \pm 7.82$ \\
& Nonhabituated-EP & $55.08 \pm 5.33$ \\
& Nonhabituated-RU & $51.07 \pm 3.40$ \\
& Nonhabituated-RU+EP & $48 \pm 3.59$ \\
\hline
\end{tabular}

Exploration times (s) of the different experimental groups in the test phase.

Data are presented as means \pm standard error of the mean.

decrease in exploratory activity as a function of repeated exposure to the same environment (Hui et al, 2005) and enhanced the exploration and novelty seeking following exposure to stress (Thorsell et al, 2006). However, no differences were found between the different treatments in the habituated and nonhabituated groups suggesting that the exposure to the stressor and the drugs had no discernible effects on locomotor activity or the normal tendency for exploration of objects.

In the following experiment, we aimed to demonstrate that when there is a delay between the training and exposure to the stressor (ie, exposure to the stressor is $3 \mathrm{~h}$ after training), the stressor will not affect performance. This will indicate that recognition memory consolidates by this time point and that the effects obtained are owing to interaction between the arousal level and the out-of-context stressor. Thus, habituated and nonhabituated animals were placed on the EP $3 \mathrm{~h}$ ( $3 \mathrm{~h} \mathrm{EP}$ ) after the sample phase or were taken to their home cage (control; Figure $3 \mathrm{~b} ; n=5$ each). A two-way ANOVA conducted on discrimination index on day 2 (arousal: habituated/nonhabituated; stress: stress/no-stress) revealed a significant effect of arousal $\left(\mathrm{F}_{(1,16)}=92.031, p<0.001\right)$, but not stress $\left(\mathrm{F}_{(1,16)}<1, \mathrm{NS}\right)$ or the interaction between arousal and stress $\left(\mathrm{F}_{(1,16)}<1, \mathrm{NS}\right)$. Further analysis of data showed a significant difference between the groups in discrimination index in the test on day $2(\mathrm{~F}(3,16)=30.82, p<0.0001)$. Post hoc comparisons showed that the habituated groups (control and stressed) spent significantly more time exploring the new object compared with the nonhabituated groups $(p<0.001)$. Thus, $3 \mathrm{~h}$ after the sample, exposure to the stressor has no effect on consolidation.

It might be argued that the poor performance of the nonhabituated animals as compared with the habituated ones, results from the different treatment they received before the training (handling instead of being exposed extensively to a context). Thus, in another experiment, we examined whether an exposure of the nonhabituated rats to another context, similar to the context in which the training takes place, would still result in poor performance. Accordingly, two groups of animals were habituated to another experimental apparatus (another open field, $50 \times 50 \times 50 \mathrm{~cm}$; context A) by allowing them to explore it for $5 \mathrm{~min}$ twice a day for 4 days before the experiment was performed.

These rats were exposed to the sample phase on day 1 and immediately afterwards were either taken to their home cage (cont, $n=7)$ or exposed to the EP stress (EP, $n=7$ ) (data not shown). $t$-Test analysis of performance during the test revealed that the stress group spent significantly more time exploring the new object as compared with the control group $(t(12)=4.473, p<0.001)$ ( $t$-test for difference from 0.5: EP: $t(6)=4.771, p<0.01)$. This suggests that in order to perform well in the test, animals should be habituated in the specific context of the arena of the task (like the habituated animals), and not in a similar context. Further, there was no significant difference between the groups in discrimination index $(t(12)<1$, NS) during the sample phase (day 1 ; data not shown). No significant differences in total exploration time were found between the groups during either the sample phase $(t(12)<1, N S)$ or the test phase $(t(12)=1.01, \mathrm{NS})$

\section{Similar Performance of Habituated and Nonhabituated Rats in the Reconsolidation of Object Recognition}

Eight groups of rats that were habituated to the experimental context before training (habituated) or just handled (nonhabituated) were exposed to the sample phase on day 1. Twenty-four hours afterwards, animals were exposed to the same two objects (reactivation) and, immediately afterwards, were either microinfused with vehicle to the BLA and taken to their home cage (habituated cont, $n=7$; nonhabituated cont, $n=7)$; exposed to the EP stress (habituated EP, $n=8$; nonhabituated EP, $n=7$ ); microinfused with RU-486 and placed on the EP (habituated RU $+\mathrm{EP}, n=70$; nonhabituated RU + EP, $n=9$ ); or microinfused with RU-486 (habituated RU, $n=6$; nonhabituated RU, $n=5$ ), with no exposure to the stressor (Figure $4 \mathrm{a}$ ).

A three-factor ANOVA conducted on discrimination index on day 3 (arousal: habituated/nonhabituated; drug: RU-486/vehicle; stress: stress/no-stress) revealed significant effects of the drug $\left(\mathrm{F}_{(1,48)}=23.266, p<0.001\right)$, and the stress $\left(\mathrm{F}_{(1,48)}=41.287, p<0.001\right)$, but not arousal $\left(\mathrm{F}_{(1,48)}<1, \mathrm{NS}\right)$. The interaction between drug and stress was significant $\left(\mathrm{F}_{(1,48)}=32.706, p<0.001\right)$, but not between arousal and drug $\left(\mathrm{F}_{(1,48)}<1, \mathrm{NS}\right)$, arousal and stress $\left(\mathrm{F}_{(1,48)}<1, \mathrm{NS}\right)$, or between arousal, drug, and stress $\left(\mathrm{F}_{(1,48)}<1\right.$, NS). Further analysis of data showed a significant difference between the groups in discrimination index in the test on day 3 $(\mathrm{F}(7,48)=14.909, p<0.0001)$. Post hoc comparisons revealed that the stressed habituated and nonhabituated groups (EP) spent significantly less time exploring the new object compared with the other groups $(p<0.0001$ for all groups) ( $t$-test for difference from 0.5: habituated: cont: $t(5)=8.863, p<0.001$; RU: $t(5)=10.142, p<0.001$; RU + EP: 

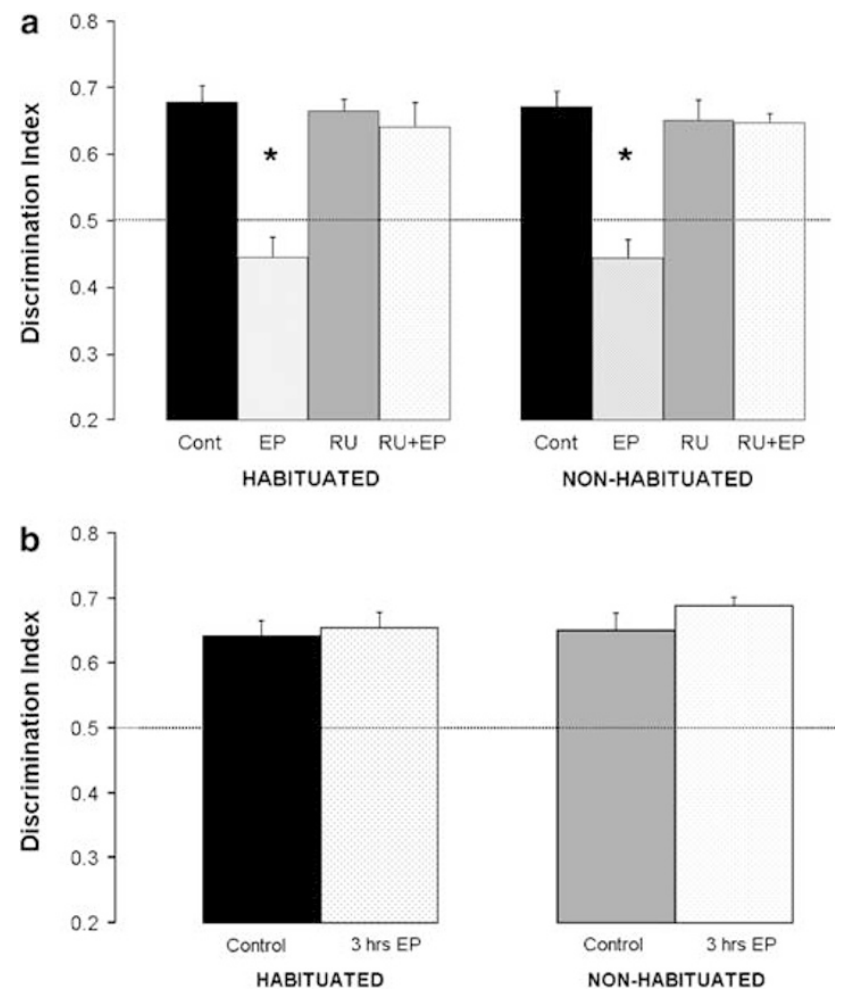

Figure 4 Similar performance of habituated and nonhabituated rats in the reconsolidation of object recognition. (a) Habituated and nonhabituated rats that were exposed to the stressor after the reactivation of the memory trace showed impaired performance in the test on day 3 compared with the other groups ( $p<0.0$ I for habituated, $p<0.00$ I for nonhabituated). In both habituated and nonhabituated rats, RU-486 into the BLA reversed the impairing effects of the stressor on performance. (b) Habituated and nonhabituated rats that were exposed to the stressor $3 \mathrm{~h}$ after the reactivation phase were no different from their control counterparts, that is, showed intact performance in the test on day 3.

$t(6)=4.204, p<0.01$; nonhabituated: cont: $t(6)=7.1223$, $p<0.001$; RU: $t(4)=7.22, p<0.01 ; \mathrm{RU}+\mathrm{EP}: t(8)=11.671$, $p<0.001)$. Thus, exposure to stress impaired reconsolidation in the habituated and nonhabituated rats, and microinfusing RU-486 to the BLA reversed the impairment. Merely microinfusing RU-486 to the BLA in the nonstressed rats did not affect their performance. Further, there was no significant difference between the groups in discrimination index during the sample phase (day $1 ; \mathrm{F}(7,48)<1$, NS; data not shown) or the reactivation phase (day $2 ; \mathrm{F}(7,48)<1$, NS; data not shown). Thus, all groups exhibited similar levels of performance during the reactivation session. No significant differences in total exploration time were found between the groups during the test phase $(\mathrm{F}(7,48)=1.945, p=0.082$; Table 1).

In the following experiment, we aimed to demonstrate that when there is a delay between the reactivation and exposure to the stressor (ie, exposure to the stressor is $3 \mathrm{~h}$ after reactivation), the stressor will not affect performance. This will indicate that recognition memory reconsolidates by this time point and that the effects obtained are owing to the interaction between the arousal level and the out-ofcontext stressor. To that end, habituated and nonhabituated animals were placed on the EP $3 \mathrm{~h}$ (EP $3 \mathrm{~h}$ ) after the reactivation phase or were taken to their home cage (control; Figure $4 \mathrm{~b} ; n=6$ each). A two-way ANOVA conducted on discrimination index on test day 3 (arousal: habituated/nonhabituated; stress: stress/no-stress) did not show significant effects of arousal $\left(\mathrm{F}_{(1,21)}<1\right.$, NS), stress $\left(\mathrm{F}_{(1,21)}=1.793, \mathrm{NS}\right)$ or the interaction between arousal and stress $\left(\mathrm{F}_{(1,21)}<1, \mathrm{NS}\right)$. Thus, $3 \mathrm{~h}$ after the reactivation, the reconsolidation window has closed.

\section{Control Experiments for the Reactivation of the Memory Trace}

The nonhabituated control rats showed intact memory in the test $24 \mathrm{~h}$ following reactivation (Figure $4 \mathrm{a}$ ). It could be argued that their good performance was a result of their exposure to the arena twice before the test (sample phase and reactivation), as opposed to only one exposure in the consolidation protocol. To verify that their good performance in the test was derived from the reactivation of the memory trace and not merely from being exposed twice to the arena, three experiments were performed. In these experiments, there is no reactivation of the memory trace, thus, we expect nonhabituated control animals to perform the task poorly, as seen in the consolidation experiment.

In the first experiment, the nonhabituated rats were exposed to the arena on day 1 (with no objects) and then exposed to two different objects for $5 \mathrm{~min}$ (sample phase) on day 2. Immediately afterwards, they were taken to their home cage (cont, $n=7$ ) or placed on an EP (EP, $n=7$; Figure 5a, day 1 Arena). $t$-Test results revealed that the control rats spent significantly less time exploring the new object compared with the stressed rats $(t(12)=5.391$, $p<0.001)$ in the test on day 3 ( $t$-test for difference from 0.5: EP: $t(6)=5.039, p<0.01)$. Thus, merely being exposed twice to the arena is not enough for intact retrieval. Further, there was no significant difference between the groups in discrimination index $(t(12)<1$, NS) during the sample phase (day 1; data not shown).

In the second experiment, nonhabituated animals were exposed to different objects on day 1 and day 2 . In the classical reconsolidation protocol, animals were exposed on day 1 and day 2 to objects $A B$, and on day 3 to the familiar object $\mathrm{A}$ and to a new object $\mathrm{C}$. In the present experiment, animals were exposed on day 1 to objects $A B$, on day 2 to objects $C D$, and on day 3 to the familiar object $C$ and to a new object E. Thus, immediately after the exposure to objects on day 2, nonhabituated rats were taken to their home cage (cont, $n=7$ ) or exposed to the EP stress (EP, $n=7$ ) (Figure $5 \mathrm{a}, \mathrm{AB}-\mathrm{CD}-\mathrm{CE}$ ). $t$-Test revealed that the stress group spent significantly more time exploring the new object compared with the control group $(t(12)=5.145$, $p<0.001$ ) in the test on day 3 ( $t$-test for difference from 0.5 : EP: $t(6)=5.99, p<0.001)$. Thus, when there was no reactivation of the memory for a familiar object on day 2 , control animals did not perform well in the test. Further, there was no significant difference between the groups in discrimination index during the sample phases on day 1 and day $2(t(12)<1$, NS; data not shown).

In the third experiment, we aimed to confirm that recognition memory is consolidated at the time of reactivation. To that end, habituated and nonhabituated animals were exposed to the sample phase on day 1. On day 2 , habituated and nonhabituated rats were exposed to the 

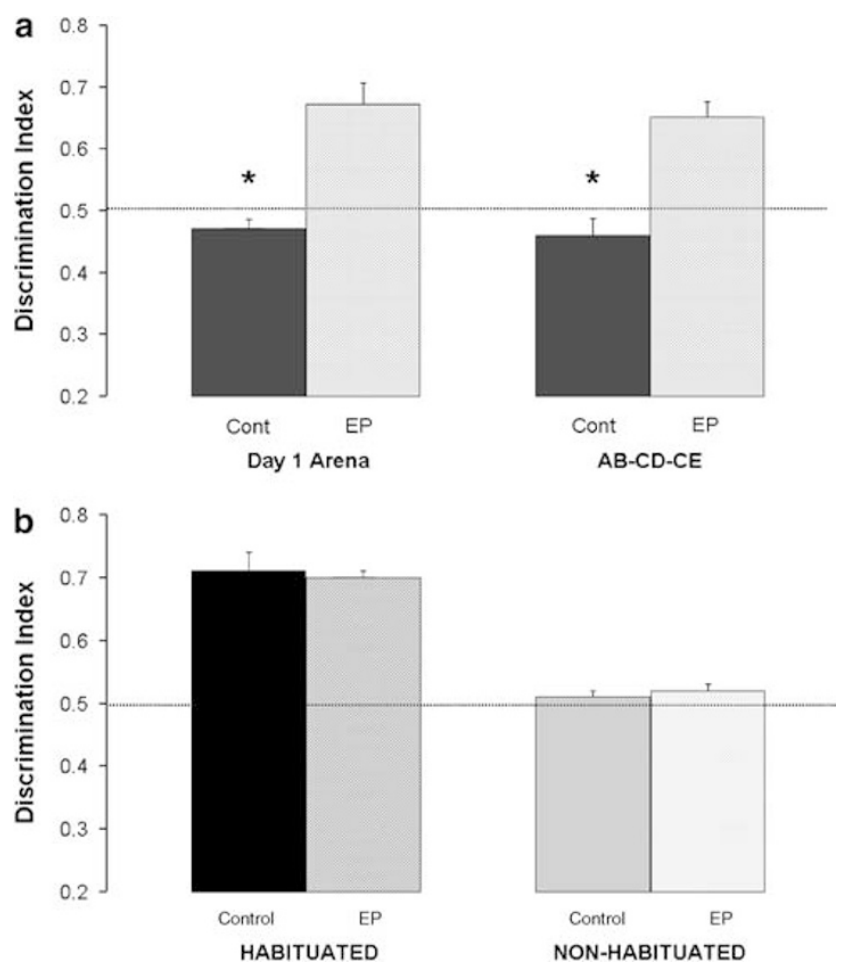

Figure 5 Reactivation of the memory trace. (a) Left: nonhabituated rats were exposed to the arena on day I (with no objects) and exposed to two different objects for $5 \mathrm{~min}$ (sample phase) on day 2. The control rats showed impaired performance in the test on day 3 compared with the stressed rats $(* p<0.00 \mathrm{I})$. Right: nonhabituated rats were exposed to different objects on day I (sample) and day 2. The control rats showed impaired performance in the test on day 3 compared with the stressed rats $(* p<0.00 \mathrm{I})$. (b) Habituated and nonhabituated rats were exposed to two different objects for $5 \mathrm{~min}$ (sample phase) on day I. On day 2, the rats were exposed to the context of the arena and were taken to their home cage or exposed to the stressor. Exposure to the stressor did not impair or facilitate performance in the habituated and nonhabituated rats, respectively.

context of the arena (with no specific reactivation of the memory trace) and, immediately afterwards, they were either taken to their home cage (habituated cont, $n=8$; nonhabituated cont, $n=7$ ) or exposed to the EP stress (habituated EP, $n=8$; nonhabituated EP, $n=7$; Figure 5b).

A two-way ANOVA conducted on discrimination index on day 3 (arousal: habituated/nonhabituated; stress: stress/no-stress) revealed a significant effect of arousal $\left(\mathrm{F}_{(1,26)}=78.017, p<0.001\right)$, but not stress $\left(\mathrm{F}_{(1,26)}<1\right.$, NS) or the interaction between arousal and stress $\left(\mathrm{F}_{(1,26)}<1, \mathrm{NS}\right)$. Further analysis of data showed a significant difference between the groups in discrimination index $(F(3,26)=$ 13.772, $p<0.001$ ) in the test on day 3. Post hoc comparisons showed that the nonhabituated control and stress groups spent significantly less time exploring the new object compared with the habituated control and stress groups $(p<0.001)$ ( $t$-test for difference from 0.5: habituated cont: $t(7)=6.642 \quad p<0.0001$; habituated EP: $t(7)=13.4$, $p<0.001)$. Importantly, the stress did not impair or facilitate performance in habituated and nonhabituated rats, respectively, and the nonhabituated control animals showed impaired performance as in the consolidation protocol. Thus, when the memory trace is not reactivated, exposure to the stressor has no effect on performance. In addition, there was no significant difference between the groups in discrimination index $(\mathrm{F}(3,26)<1, \mathrm{NS})$ during the sample phase (day 1).

\section{Beta-Adrenoceptors in the BLA Mediate the Performance of Nonhabituated Rats in the Consolidation, but Not in the Reconsolidation, of Object Recognition Memory}

In the next experiment, we sought to examine whether the poor performance of the nonhabituated control rats in the consolidation test would be reversed by a $\beta$-adrenoceptor blocker into the BLA. Immediately after the sample phase, nonhabituated rats were either microinfused with saline to the BLA and taken to their home cage (cont); exposed to the EP stress (EP); microinfused with Prop and placed on the EP (Prop +EP); or microinfused with Prop, with no exposure to the stressor (Figure $6 \mathrm{a} ; n=7$ each). A twoway ANOVA conducted on discrimination index on day 2 (drug: prop/vehicle; stress: stress/no-stress) revealed significant effects of drug $\left(\mathrm{F}_{(1,24)}=8.949, p=0.006\right)$, and the interaction between the drug and the stress $\left(\mathrm{F}_{(1,24)}=24.433\right.$, $p<0.001)$, but not stress $\left(\mathrm{F}_{(1,24)}=2.382\right.$, NS). Further analysis of data showed a significant difference between the groups in discrimination index $(\mathrm{F}(3,27)=13.772$, $p<0.001)$ in the test on day 2. Post hoc comparisons revealed that the control group spent significantly less time exploring the new object compared with the other groups (EP and Prop + EP: $p<0.001$, Prop: $p<0.001$ ) ( $t$-test for difference from 0.5: EP: $t(6)=7.53 p<0.001$; Prop: $t(6)=7.405, \quad p<0.001$; Prop + EP: $t(6)=5.148, p<0.01)$. Furthermore, a significant difference was found between the Prop +EP and the Prop group $(p<0.05)$. Thus, microinfusing Prop to the BLA after the sample phase reversed the poor performance of the nonhabituated rats in the test on the next day. In addition, there was no significant difference between the groups in discrimination index $(F(3,27)<1, N S)$ during the sample phase (day 1$)$. No significant differences in total exploration time were found between the groups during either the sample phase $(\mathrm{F}(3,27)<1, \mathrm{NS})$ or the test phase $(\mathrm{F}(3,27)<1, \mathrm{NS})$.

Next, we examined whether the $\beta$-adrenoceptor blocker would affect the good performance of the nonhabituated rats in the reconsolidation protocol. Immediately after the reactivation of the memory trace, rats were either microinfused with vehicle (cont), or Prop to the BLA and taken to their home cage (Figure $6 \mathrm{~b} ; n=7$ each). $t$-Test did not reveal a significant difference between the groups in discrimination index during the sample phase (day 1; $t(12)<1$, NS; data not shown), the reactivation phase (day 2 ; $t(12)<1$, NS; data not shown), or the test (day 3 ; $t(12)<1$, NS). Both groups performed the task well in the test ( $t$-test for difference from 0.5: cont: $t(6)=7.159, p<0.001$; Prop: $t(6)=10.934, p<0.001)$. No significant differences in total exploration time were found between the groups during the sample phase $(t(12)<1$, NS), the reactivation phase $(t(12)<1$, NS), or the test phase $(t(12)<1$, NS).

\section{DISCUSSION}

The results of this research showed that the novelty stress/ arousal experienced by the rats during training dissociated 

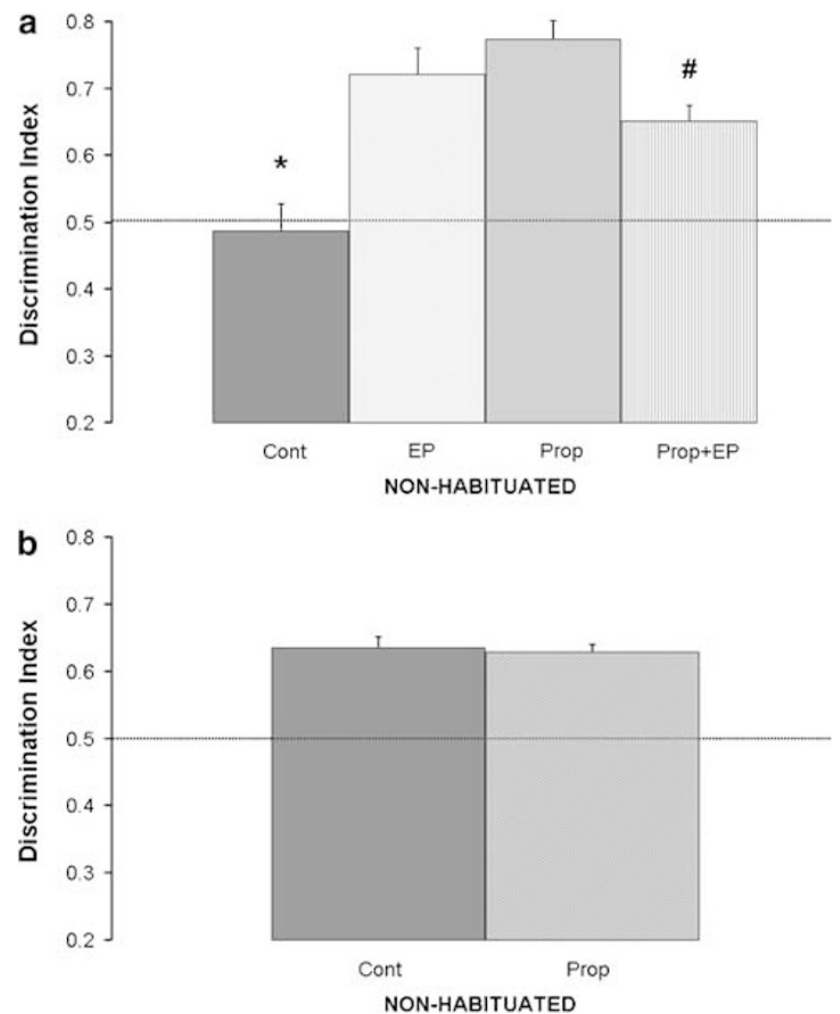

Figure 6 The involvement of $\beta$-adrenoceptors in the BLA in the consolidation and reconsolidation of object recognition of nonhabituated rats. (a) Nonhabituated control rats showed impaired performance in the test on day 2 compared with the other groups $\left(* p<0.01\right.$; ${ }^{*} p<0.05$ indicates a difference between Prop nonhabituated and Prop + Stress nonhabituated). Prop into the BLA reversed the impaired performance of nonhabituated control rats in the test. (b) Prop into the BLA of nonhabituated rats after the reactivation of the memory trace did not affect their performance in the task. Thus, the intact performance of the nonhabituated rats in the reconsolidation protocol is probably not mediated by beta-adrenoceptors in the BLA.

consolidation from reconsolidation of recognition memory. Accordingly, rats with no previous habituation to the experimental context (ie, highly aroused) demonstrated impaired performance when consolidation was examined, and good reconsolidation memory when the memory trace was reactivated. The impairment in consolidation in nonhabituated rats was found to be mediated by betaadrenoceptors in the BLA, as the Prop microinfused to the BLA immediately after training resulted in intact consolidation. Prop microinfused to the BLA immediately after the reactivation of the memory trace did not affect reconsolidation, thus, further supporting the dissociation effect. In contrast, rats that had reduced novelty stress/arousal because of extensive prior habituation performed well in both consolidation and reconsolidation memory tests.

Our data further demonstrate that exposure to an out-ofcontext stressor facilitated memory consolidation, but impaired reconsolidation in the nonhabituated rats. However, in the habituated rats, the stressor impaired both consolidation and reconsolidation of recognition memory. A GR antagonist infused into the BLA reversed the impairing as well as the facilitating effects of the stressor on memory in both the habituated and the nonhabituated rats. Thus, GR mechanism in the BLA might potentially explain the effects of the stressor on recognition memory. It should be noted that although we used a small infusion volume ( $0.5 \mu$ l volume per side), the drugs may have spread to adjacent areas, such as the central nucleus of the amygdala.

Importantly, we showed that when we placed habituated and nonhabituated animals on the EP $3 \mathrm{~h}$ after the sample or the reactivation of the memory trace, there was no effect on either consolidation or reconsolidation of memory. Thus, the exposure to the stressor should be within the time window of consolidation and reactivation to affect the memory trace. This confirms that recognition memory is consolidated at the time of reactivation and it corresponds to previously reported data that show that reconsolidation could be manipulated only following reactivation of the specific memory trace (Akirav and Maroun, 2006; Nader et al, 2000a).

\section{Differential Effects of Arousal Level on Recognition Memory}

The emotional state of the animal during training has a considerable effect on memory, as seen by the dissimilar performance of the habituated and the nonhabituated rats. The response to novelty is a complex mechanism that involves several processes, including arousal, attention, anxiety, and stress-related factors. Arousal and attention are necessary when the animal is exposed to a novel environment in order to get acquainted with it (Cerbone and Sadile, 1994; Hui et al, 2005; Thiel et al, 1998). The open-field test showed that the nonhabituated rats spent significantly less time exploring the center of the open-field arena as compared with the habituated rats, indicating a higher level of anxiety. Furthermore, nonhabituated animals showed twofold increase in their total exploration time in the arena compared with the habituated animals. This supports other studies suggesting a decrease in exploratory activity as a function of repeated exposure to the same environment (Hui et al, 2005) and enhanced exploration and novelty seeking following the exposure to stress (Thorsell et al, 2006). Taken together, these findings corroborate those of other studies showing that nonhabituated animals are emotionally different from their habituated counterparts (Cerbone and Sadile, 1994; Okuda et al, 2004). It is important to note that there was no difference in total exploration time between controls and animals that were exposed to the EP within the habituated and nonhabituated groups. Thus, the stressor did not have a notable effect on the animals' exploration time and thus it opposes the argument that the exposure to the stressor might have confounded with the object recognition task.

It has been reported (Okuda et al, 2004) that nonhabituated animals have higher levels of corticosterone than habituated animals. Additionally, post-training corticosterone injections enhanced the performance of the nonhabituated rats in a test given $24 \mathrm{~h}$ after training. This corresponds with our finding of an enhancing effect of stress in nonhabituated rats. Why is it that the nonhabituated rats do not learn? We suggest that the arousal/stress induced by the novel context impairs their memory. The most apparent difference seen between habituated and nonhabituated animals is their total 
exploration time during the test and sample. Although nonhabituated animals spent more time exploring the two objects, their consolidation was impaired. This is surprising as in the recognition task, more total exploration time results in strong novel object preference. For example, several studies set a criterion for minimal exploration time that has to be achieved by all the animals (eg until it had accumulated $30 \mathrm{~s}$ or more of object exploration; Ainge et al, 2006; Hammond et al, 2004). Furthermore, nonhabituated animals showed high levels of exploration when reconsolidation was tested and their performance in this task was intact. Taken together, these suggest that the difference between habituated and nonhabituated animals cannot be attributed to differences in total exploration time.

\section{Dissociation of Consolidation from Reconsolidation of Recognition Memory as a Function of the Novelty Arousal Experienced during Training}

The differentiation between the performance of nonhabituated rats in the consolidation and the reconsolidation of recognition memory seems to be dependent on the arousal level experienced by the animal and mediated by adrenoceptors in the BLA. It has been proposed that memories require intact beta-receptors to reconsolidate the memory trace after use (Sara, 2000). Prop, administered after reactivation, has been shown to impair the reconsolidation of a positively reinforced radial maze task, as well as passive avoidance and fear conditioning (Debiec and LeDoux, 2004; Przybyslawski et al, 1999). In our study, Prop into the BLA after reactivation did not affect the performance of the nonhabituated rats. Perhaps a systemic microinfusion would have resulted in a different outcome, and this should be addressed in future studies. In a recent paper (Roozendaal et al, 2006), it has been reported that endogenous noradrenergic activation of the BLA induced by the emotional arousal of nonhabituated animals is essential in enabling glucocorticoid enhancement of recognition memory. This supports our argument that the arousal level of nonhabituated animals is mediated by noradrenergic mechanisms in the BLA. Furthermore, it may suggest that the enhancing effect of the out-of-context stressor on memory consolidation in nonhabituated animals is because of an interaction between noradrenergic and glucocorticoid factors in the BLA. Nevertheless, the involvement of the beta-adrenergic blocker in the consolidation, but not the reconsolidation, of the object recognition task further supports our claim that there is a dissociation of consolidation from reconsolidation that may be explained by the arousal level of the animal during the training.

Our dissociation effect is in line with others suggesting dissimilarities between consolidation and reconsolidation. For example, protein synthesis and $\mathrm{C} / \mathrm{EBP} \beta$ in the dorsal hippocampus are crucial for consolidation, but not for reconsolidation of inhibitory avoidance memory (Taubenfeld et al, 2001). Similarly, a double dissociation between consolidation and reconsolidation in the hippocampus was found with brain-derived neurotrophic factor and zif268 (Lee et al, 2004).

Prop to the BLA or exposure to a stressor had the same effect of enhancing consolidation of nonhabituated control rats. It is interesting why microinfusing Prop to the BLA and then exposing the animals to a stressor resulted in not as much of an enhancement as Prop induced by itself. The actions of Prop to reduce arousal may have interacted with the effects of stress, but we cannot infer on the basis of this result whether the actions of Prop to reduce arousal and stress are unitary or dissociable.

\section{Opposite Effect of the Stressor on the Consolidation of Recognition Memory in Habituated and Nonhabituated Animals}

Considerable evidence indicates enhancing as well as impairing effects following acute stress or acute glucocorticoid treatment (de Kloet et al, 1999; Lupien and McEwen, 1997; Roozendaal, 2002). The finding that memory consolidation was enhanced in nonhabituated rats following exposure to the stressor corresponds with studies showing that if an animal learns a stressful task, then the consolidation of this task may be enhanced by stress and that its end product, corticosterone, may be secreted during the task (de Kloet et al, 1999, 1998; Roozendaal, 2003). Thus, the level of stress applied in our experiment is apparently in the range that provokes a facilitation of memory retention. This was found to be the case in a variety of emotionally arousing tasks, such as inhibitory avoidance, spatial learning, discrimination learning, and fear conditioning (Cordero and Sandi, 1998; Flood et al, 1978; Hui et al, 2004; Kovacs et al, 1977; Pugh et al, 1997; Roozendaal and McGaugh, 1996; Sandi et al, 1997).

However, it seems that the enhancing effect is more relevant to aversive tasks (Wolf, 2003) than to nonaversive tasks, as the stressor was found to impair the consolidation of object recognition in the habituated rats. Repeated habituation is known to reduce the novelty-induced arousal response, and under these circumstances, stress can have detrimental effects on memory. There are reports that if an animal is placed in a stressful environment immediately following training, performance in delayed recall may be impaired (Cabib and Castellano, 1997; Diamond et al, 2006; Klenerova et al, 2003; Meere and Borrell, 1988; Rudy, 1996; Trneckova et al, 2005). Overall, these findings suggest that the level of arousal or stress experienced by the animal during training is an important factor in determining whether stress will enhance or impair memory consolidation. Yet, another explanation may be that a stressor may enhance memory consolidation in animals with otherwise poor memory whereas the same stressor impairs memory if the controls already show good memory. This phenomenon is known for drug effects on memory consolidation and forms the foundation of the inverted-U-shaped doseresponse effect.

Interestingly, in the habituated group, exposure to the stressor increased the rats' tendency to explore the old object. One possible explanation is that exposure to the stressor disrupted the consolidation and animals exhibited impaired retrieval as they did not remember the familiar object they have seen before. Yet another possible explanation is that the animals have associated the aversiveness of the stressor with what preceded its exposure to the stressor (ie, the arena). Once placed again in the arena for the test, rats explored the familiar object more 
than the novel object because they wanted to avoid novelty, and this was expressed in avoiding the novel object and preferring the familiar one that signals safety. This does not necessarily suggest that they had an explicit memory of the old object, but that the stressor somehow changed the emotional state of the rats such that they will avoid novelty. Thus, the exploration of the old object may be mediated by familiarity and not by explicit memory.

\section{The Stressor Impairs the Reconsolidation of Recognition Memory}

Both habituated and nonhabituated control rats performed well in the task following reactivation of the memory trace. Thus, it seems that the reactivation of the memory trace contributed to stabilizing the original memory of the objects. We tend to rule out that this strengthening of the memory trace might result from the mere exposure to the arena, as the nonhabituated rats that were exposed to the arena twice before the test (with one exposure to the objects) (Figure 5a, left), or merely exposed to the context of the arena (Figure $5 b$ ) showed impaired performance.

Furthermore, exposing nonhabituated animals to different objects in the arena twice before the test (and thus without a specific reactivation of the memory trace; Figure 5a, right) also resulted in poor performance in the task. Hence, we suggest that nonhabituated animals are still highly aroused after two exposures to the arena and objects and that this arousal has an impairing effect on the consolidation of the memory trace, but not its reconsolidation.

\section{The Role of GRs in Mediating the Effects of Stress in the BLA}

Extensive evidence focuses on the role of the amygdala, which expresses a moderate density of GRs (Honkaniemi et al, 1992; Morimoto et al, 1996), as a critical system that mediates stress hormone effects on memory consolidation (McGaugh, 2000). The BLA seems to be a key structure in a memory-modulatory system that regulates stress and glucocorticoid effects on memory consolidation, memory retrieval, and working memory (Roozendaal, 2002, 2003). The results of the present study suggest that stress effects on memory consolidation depend on post-training activation of glucocorticoid-sensitive pathways. Glucocorticoids readily enter the brain and bind directly to mineralocorticoid receptors (MRs) and GRs (Reul and de Kloet, 1985). The two receptor types differ in their affinity for corticosterone and synthetic ligands, with MRs having a higher affinity for the natural steroids and GRs for the synthetic steroids (Reul and de Kloet, 1985; Reul et al, 1990). Several findings suggest that the memory-modulating effects of glucocorticoids selectively involve activation of GRs and not MRs (de Kloet et al, 1998; Oitzl and de Kloet, 1990; Sandi and Rose, 1994a,b). The GR antagonist has been shown to enhance memory in several aversive tasks (Roozendaal and McGaugh, 1997a, b). Here, however, merely infusing a GR antagonist to the BLA did not affect performance in the control animals. Therefore, the basic differences in performance between the habituated and the nonhabituated rats cannot be explained through GR mechanisms in the amygdala.

\section{Summary}

A major conclusion from this study is that although some general features underlie consolidation and reconsolidation, there is a possible dissimilarity between the two processes, which is dependent on the emotional state of the animal during training. It has been proposed that reactivating traumatic memories under conditions that would prevent reconsolidation may help in treating post-traumatic stress disorder (PSTD) or other anxiety-related conditions (Nader, 2003). We present first evidence, as far as we know, for the deleterious effects that a stressful experience might have on the reconsolidation of a memory trace. Thus, this raises an interesting possibility that the stress that is induced by recalling the traumatic episode might affect its own reconsolidation or that part of the symptomatology in PTSD might result from the fact that the trauma impairs the reconsolidation of any memories that are active at the time.

\section{ACKNOWLEDGEMENTS}

Supported by a grant from The National Institute for Psychobiology in Israel (no. 22-2004-5) to MM, by The Ebelin and Gerd Bucerius ZEIT Foundation to MM, and by a postdoctoral fellowship from The National Institute for Psychobiology in Israel to IA. We thank Larisa Pershin and Noam Hikind for technical help.

\section{REFERENCES}

Ainge JA, Heron-Maxwell C, Theofilas P, Wright P, de Hoz L, Wood ER (2006). The role of the hippocampus in object recognition in rats: examination of the influence of task parameters and lesion size. Behav Brain Res 15: 183-195.

Akirav I, Maroun M (2006). Ventromedial prefrontal cortex is obligatory for consolidation and reconsolidation of object recognition memory. Cereb Cortex 16: 1759-1765.

Akirav I, Richter-Levin G (2002). Mechanisms of amygdala modulation of hippocampal plasticity. J Neurosci 15: 9912-9921.

Akirav I, Sandi C, Richter-Levin G (2001). Differential activation of hippocampus and amygdala following spatial learning under stress. Eur J Neurosci 14: 719-725.

Alberini CM (2005). Mechanisms of memory stabilization: are consolidation and reconsolidation similar or distinct processes? Trends Neurosci 28: 51-56.

Anokhin KV, Tiunova AA, Rose SP (2002). Reminder effectsreconsolidation or retrieval deficit? Pharmacological dissection with protein synthesis inhibitors following reminder for a passive-avoidance task in young chicks. Eur J Neurosci 15: 1759-1765.

Cabib S, Castellano C (1997). Impairments produced by amphetamine and stress on memory storage are reduced following a chronic stressful experience. Psychopharmacology (Berl) 129: 161-167.

Cerbone A, Sadile AG (1994). Behavioral habituation to spatial novelty: interference and noninterference studies. Neurosci Biobehav Rev 18: 497-518.

Cordero MI, Sandi C (1998). A role for brain glucocorticoid receptors in contextual fear conditioning: dependence upon training intensity. Brain Res 786: 11-17.

de Kloet ER, Oitzl MS, Joels M (1999). Stress and cognition: are corticosteroids good or bad guys? Trends Neurosci 22: 422-426.

de Kloet ER, Vreugdenhil E, Oitzl MS, Joels M (1998). Brain corticosteroid receptor balance in health and disease. Endocr Rev 19: $269-301$. 
Debiec J, LeDoux JE (2004). Disruption of reconsolidation but not consolidation of auditory fear conditioning by noradrenergic blockade in the amygdala. Neuroscience 129: 267-272.

Debiec J, LeDoux JE, Nader K (2002). Cellular and systems reconsolidation in the hippocampus. Neuron 24: 527-538.

Diamond DM, Campbell AM, Park CR, Woodson JC, Conrad CD, Bachstetter AD et al (2006). Influence of predator stress on the consolidation versus retrieval of long-term spatial memory and hippocampal spinogenesis. Hippocampus 16: 571-576.

Diamond DM, Fleshner M, Ingersoll N, Rose GM (1996). Psychological stress impairs spatial working memory: relevance to electrophysiological studies of hippocampal function. Behav Neurosci 110: 661-672.

Dudai Y (2002). Molecular bases of long-term memories: a question of persistence. Curr Opin Neurobiol 12: 211-216.

Durand M, Berton O, Aguerre S, Edno L, Combourieu I, Mormede $\mathrm{P}$ et al (1999). Effects of repeated fluoxetine on anxiety-related behaviours, central serotonergic systems, and the corticotropic axis in SHR and WKY rats. Neuropharmacology 38: 893-907.

Eisenberg M, Kobilo T, Berman DE, Dudai Y (2003). Stability of retrieved memory: inverse correlation with trace dominance. Science 22: 1102-1104.

Ennaceur A, Delacour J (1988). A new one-trial test for neurobiological studies of memory in rats. 1. Behavioral data. Behav Brain Res 1: 47-59.

Flood JF, Vidal D, Bennett EL, Orme AE, Vasquez S, Jarvik ME (1978). Memory facilitating and anti-amnesic effects of corticosteroids. Pharmacol Biochem Behav 8: 81-87.

Hammond RS, Tull LE, Stackman RW (2004). On the delaydependent involvement of the hippocampus in object recognition memory. Neurobiol Learn Mem 82: 26-34.

Honkaniemi J, Pelto-Huikko M, Rechardt L, Isola J, Lammi A, Fuxe K et al (1992). Colocalization of peptide and glucocorticoid receptor immunoreactivities in rat central amygdaloid nucleus. Neuroendocrinology 55: 451-459.

Hui GK, Figueroa IR, Poytress BS, Roozendaal B, McGaugh JL, Weiberger NM (2004). Memory enhancement of classical fear conditioning by post-training injections of corticosterone in rats. Neurobiol Learn Mem 81: 67-74.

Hui Z, Guang-Yu M, Chong-Tao X, Quan Y, Xiao-Hu X (2005). Phenytoin reverses the chronic stress-induced impairment of memory consolidation for water maze training and depression of LTP in rat hippocampal CA1 region, but does not affect motor activity. Cogn Brain Res 24: 380-385.

Izumi J, Washizuka M, Hayashi-Kuwabara Y, Yoshinaga K, Tanaka Y, Ikeda Y et al (1997). Evidence for a depressive-like state induced by repeated saline injections in Fischer 344 rats. Pharmacol Biochem Behav 57: 883-888.

Kavushansky A, Richter-Levin G (2006). Effects of stress and corticosterone on activity and plasticity in the amygdala. J Neurosci Res 84: 1580-1587.

Kida S, Josselyn SA, de Ortiz SP, Kogan JH, Chevere I, Masushigh S et al (2002). CREB required for the stability of new and reactivated fear memories. Nat Neurosci 5: 348-355.

Klenerova V, Jurcovicova J, Kaminsky O, Sida P, Krejci I, Hlinal Z et al (2003). Combined restraint and cold stress in rats: effects on memory processing in passive avoidance task and on plasma levels of ACTH and corticosterone. Behav Brain Res 142: 143-149.

Kovacs GL, Telegdy G, Lissak K (1977). Dose-dependent action of corticosteroids on brain serotonin content and passive avoidance behavior. Horm Behav 8: 155-165.

Lee JL, Everitt BJ, Thomas KL (2004). Independent cellular processes for hippocampal memory consolidation and reconsolidation. Science 7: 839-843.

Lupien SJ, McEwen BS (1997). The acute effects of corticosteroids on cognition: integration of animal and human model studies. Brain Res Rev 24: 1-27.
Maroun M, Richter-Levin G (2003). Exposure to acute stress blocks the induction of long-term potentiation of the amygdalaprefrontal cortex pathway in vivo. J Neurosci 1: 4406-4409.

McGaugh JL (2000). Memory - a century of consolidation. Science 14: $248-251$.

McGaugh JL (2002). Memory consolidation and the amygdala: a systems perspective. Trends Neurosci 25: 456.

McIntyre CK, Power AE, Roozendaal B, McGaugh JL (2003). Role of the basolateral amygdala in memory consolidation. Ann NY Acad Sci 985: 273-293.

Meere M, Borrell J (1988). Psychoneurogenic stress influences upon retention of an inhibitory avoidance response in the rat. Behav Neural Biol 49: 1-11.

Milekic MH, Alberini CM (2002). Temporally graded requirement for protein synthesis following memory reactivation. Neuron 24: 521-525.

Morimoto M, Morita N, Ozawa H, Yokoyama K, Kawata M (1996). Distribution of glucocorticoid receptor immunoreactivity and mRNA in the rat brain: an immunohistochemical and in situ hybridization study. Neurosci Res 26: 235-269.

Nader K (2003). Memory traces unbound. Trends Neurosci 26: 65-72.

Nader K, Schafe GE, LeDoux JE (2000a). Fear memories require protein synthesis in the amygdala for reconsolidation after retrieval. Nature 17: 722-726.

Nader K, Schafe GE, LeDoux JE (2000b). The labile nature of consolidation theory. Nat Rev Neurosci 1: 216-219.

Oitzl MS, de Kloet ER (1990). Selective corticosteroid antagonists modulate specific aspects of spatial orientation learning. Behav Neurosci 106: 62-71.

Okuda S, Roozendaal B, McGaugh JL (2004). Glucocorticoid effects on object recognition memory require trainingassociated emotional arousal. Proc Natl Acad Sci USA 101: 853-858.

Pare WP (1994). Open field, learned helplessness, conditioned defensive burying, and forced-swim tests in WKY rats. Physiol Behav 55: 433-439.

Przybyslawski J, Roullet P, Sara SJ (1999). Attenuation of emotional and nonemotional memories after their reactivation: role of beta adrenergic receptors. J Neurosci 1: 6623-6628.

Przybyslawski J, Sara SJ (1997). Reconsolidation of memory after its reactivation. Behav Brain Res 84: 241-246.

Pugh CR, Tremblay D, Fleshner M, Rudy JW (1997). A selective role for corticosterone in contextual-fear conditioning. Behav Neurosci 111: 503-511.

Ramos A, Berton O, Mormede P, Chaouloff F (1997). A multipletest study of anxiety-related behaviours in six inbred rat strains. Behav Brain Res 85: 57-69.

Reul JM, de Kloet ER (1985). Two receptor systems for corticosterone in rat brain: microdistribution and differential occupation. Endocrinol 117: 2505-2511.

Reul JM, Sutanto W, van Eekelen JA, Rothuizen J, de Kloet ER (1990). Central action of adrenal steroids during stress and adaptation. Adv Exp Med Biol 274: 243-256.

Robbins TW (1997). Arousal systems and attentional processes. Biol Psychol 45: 57-71.

Roozendaal B (2002). Stress and memory: opposing effects of glucocorticoids on memory consolidation and memory retrieval. Neurobiol Learn Mem 78: 578-595.

Roozendaal B (2003). Systems mediating acute glucocorticoid effects on memory consolidation and retrieval. Prog Neuropsychopharmacol Biol Psychiatry 27: 1213-1223.

Roozendaal B, McGaugh JL (1996). Amygdaloid nuclei lesions differentially affect glucocorticoid-induced memory enhancement in an inhibitory avoidance task. Neurobiol Learn Mem 65: $1-8$.

Roozendaal B, McGaugh JL (1997a). Basolateral amygdala lesions block the memoryenhancing effect of glucocorticoid adminis- 
tration in the dorsal hippocampus of rats. Eur J Neurosci 9: $76-83$.

Roozendaal B, McGaugh JL (1997b). Glucocorticoid receptor agonist and antagonist administration into the basolateral but not central amygdala modulates memory storage. Neurobiol Learn Mem 67: 176-179.

Roozendaal B, Okuda S, Van der Zee EA, McGaugh JL (2006). Glucocorticoid enhancement of memory requires arousalinduced noradrenergic activation in the basolateral amygdala. Proc Natl Acad Sci USA 25: 6741-6746.

Roozendaal B, Williams CL, McGaugh JL (1999). Glucocorticoid receptor activation in the rat nucleus of the solitary tract facilitates memory consolidation: involvement of the basolateral amygdala. Eur J Neurosci 11: 1317-1323.

Rudy JW (1996). Postconditioning isolation disrupts contextual conditioning: an experimental analysis. Behav Neurosci 110: 238-246.

Sandi C, Loscertales M, Guaza C (1997). Experience-dependent facilitating effect of corticosterone on spatial memory formation in the water maze. Eur J Neurosci 9: 637-642.

Sandi C, Rose SP (1994a). Corticosterone enhances long-term retention in one-day-old chicks trained in a weak passive avoidance learning paradigm. Brain Res 647: 106-112.

Sandi C, Rose SP (1994b). Corticosteroid receptor antagonists are amnestic for passive avoidance learning in day-old chicks. Eur J Neurosci 6: 1292-1297.
Sara SJ (2000). Retrieval and reconsolidation: toward a neurobiology of remembering. Learn Mem 7: 73-84.

Taubenfeld SM, Milekic MH, Monti B, Alberini CM (2001). The consolidation of new but not reactivated memory requires hippocampal C/EBPbeta. Nat Neurosci 4: 813-818.

Thiel CM, Huston JP, Schwarting RK (1998). Hippocampal acetylcholine and habituation learning. Neuroscience 85: 1253-1262.

Thorsell A, Slawecki CJ, El Khoury A, Mathe AA, Ehlers CL (2006). The effects of social isolation on neuropeptide $Y$ levels, exploratory and anxiety-related behaviors in rats. Pharmacol Biochem Behav 83: 28-34.

Torras-Garcia M, Lelong J, Tronel S, Sara SJ (2005). Reconsolidation after remembering an odor-reward association requires NMDA receptors. Learn Mem 12: 18-22.

Trneckova L, Hynie S, Sida P, Hlinak Z, Krejci I, Klenerova V (2005). Effects of stress and of amphetamine on passive avoidance conditioning in rats. Gen Physiol Biophys 24: $129-142$.

Wolf OT (2003). HPA axis and memory. Best Pract Res Clin Endocrinol Metab 17: 287-299.

Xu L, Holscher C, Anwyl R, Rowan MJ (1998). Glucocorticoid receptor and protein/RNA synthesis-dependent mechanisms underlie the control of synaptic plasticity by stress. Proc Natl Acad Sci USA 17: 3204-3208. 